nuclei and that they move electro-cataphoretically, the size of a nucleus ${ }^{9}$ being less than $10^{-6} \mathrm{~cm}$., the ratio charge to mass being sufficiently large. Even if the particle size were larger than in the above case, it may still be expected that cataphoresis will occur if their surfaces are fresh and the electric field is strong enough. In order to test this expectation, freshly deposited potassium chloride crystallites were allowed to sediment in the supersaturated solution. An external electric field was applied momentarily to the solution (between two carbon rods in the container, $150 \mathrm{~V} . / \mathrm{cm}$.) immediately after the appearance of the crystallites. The sedimentation paths were deflected a little to the cathode, though the situation was somewhat blurred, probably by the heterogeneity in the dimensions and forms of crystallites and also by the thermal convection accompanying the change of phase.

In short, all these observations support the view put forward by Ubbelohde.

Chemical Laboratory,

\section{Koichi Hirano}

Fukushima University,

Fukushima, Japan. F'eb. 12.

I Ubbelohde, A. R., Trans. Farad. Soc., 36, 863 (1940); Discuss. Farad. Soc., 5, 180 (1949).

2 Boerboom, A. J. H., Nature, 159, 230 (1947)

'Weyl, W. A., Glass Indust., 28, 231 (1947).

4 Erlenmeyer, H., Helv. Chim. Acta, 10, 896 (1927). Hazlehurst Erlenmeyer, H., Helv. Chim. Acta, 10, 896
and Martin, J. Phys. Chem., 40, 439 (1936).

'Tominaga, H., and Hirano, K., J. Chem. Soc. Japan, 73, 522 (1952).

- Hirano, K., Kagaku (Science), 23, 30 (1953).

' Kruyt, H. A., Koll. Z., 22, 81 (1918).

${ }^{8}$ Hirano, K., J. Chem. Soc. Japan, 74, 701 (1953)

${ }^{8}$ Hirano, K., Busseiron-Kenkyu, 58, 35 (1953).

RECENT observations on the crystallization of lithium sulphate by Helen Rae and A. E. Robinson may possibly provide a further example of the formation of crystallization nuclei bearing a net charge with regard to the solution. ${ }^{1}$. Attention may be directed to the interest of this mechanism of migration of nuclei of ionic crystals. With reference to the deposition of crystalline masses at controlled places in animal systems, it is conceivable that the formation of bones and teeth, for example, are controlled by the direction of nuclei from supersaturated solutions through the intervention of the appropriato electric fields in the surrounding system. The deposition of crystals associated with cortain diseases may also be related with the phenomenon under discussion.

The Queen's University,

\section{A. R. UbBelohde}

Belfast.

${ }^{2}$ Rae, Helen, and Robinson, A. E., Proc. Roy. Soc., A, 222, 558 (1954).

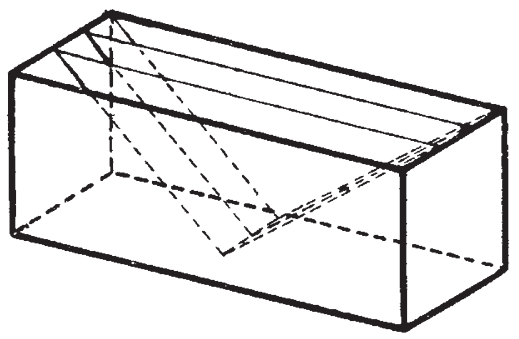

Fig. 1

I have modified this double cell in such a way that part of the parallel beam traverses only the outer cell, passing beside or under the hollow prism. I have thus obtained simultaneously the undeflected and the deflected image of the slit in the measuring microscope ; the difference may be read directly in a single measurement without having to change the contents of the hollow glass prism. Errors caused by mechanical derangement or variation of temperature during the change are thus avoided.

A further modification operates with a horizontal slit instead of the usual vertical one, and the rectangular cell contains two hollow prisms instead of one, resting with their apex cdge on the bottom of the cell (Fig. 1). Each prism occupies a third of the vertical transverse section of the rectangular cells, leaving a third free for the light beam to bypass them. The rectangular cell is filled with solvent, one of the hollow prisms with the solution to be measured and the other one with a solution of known refractive index for calibration. Thus the difference to be measured and the calibration value may be read simultaneously in one measurement. The fact that the hollow prisms stand on their apex edges makes it possible to use them with a small volume of solution, solely at the cost of light intensity owing to the reduced aperture available.

Solutions may be made up in this refractometer to a given value of difference in refractive indices by filling an appropriate solution into the calibration prism and diluting the other one with solvent from, for example, a burette until the lines-now horizontal -coincide in the microscope.

I wish to acknowledge the help of Mr. F. Dullion and Mr. J. Szántó in the construction of the new cell.

Délibáb utca 28 ,

F. KöRÖSY

Budapest.

Feb. 23.

1 Martens, J.. and Stuart, H. A., Arch. Tech. Messen., 111 (May 1853). :Debye, P. P., J. App. Phys., 17, 392 (1946).

s Brice, B. A., and Speiser, R., J. Opt. Soc. Amer., 38, 363 (1946). Brice, B., A., and Halwer, J. Opt. Soc. Amer., 41, 1033 (1951).

\section{Structure of Collagen}

A DETAILED X-ray study of collagen fibres obtained from different sources (namely, shark ray, rat tail tendon and kangaroo tail tendon) and a re-examination of the published wide-angle patterns indicate that the unit cell of collagen is hexagonal with $a=12-16 \mathrm{~A}$. and $c=9 \cdot 5-9 \mathrm{~A}$., the actual values depending on the moisture content. The essential difference of this indexing from those reported earlier ${ }^{1-3}$ is that the $2 \cdot 86$-A. meridional are is here interpreted not as a true meridional reflexion, but as arising from the superposition of two close non- 